\title{
The impact of molecular genetic analysis of the $V H L$ gene in patients with haemangioblastomas of the central nervous system
}

Sven Gläsker, Bernhard U Bender, Thomas W Apel, Ernst Natt, Vera van Velthoven, Rudolf Scheremet, Josef Zentner, Hartmut P H Neumann

\begin{abstract}
Objectives-Haemangioblastoma of the CNS occurs as a sporadic entity and as a manifestation of the autosomal dominant von Hippel-Lindau disease with the major additional components retinal angioma, renal cancer, and pheochromocytoma. Genetic testing for germline mutations predisposing to von Hippel-Lindau disease has been available since identification of the $V H L$ tumour suppressor gene. The impact of this testing was evaluated in patients with haemangioblastomas seen in this centre.
\end{abstract}

Methods-A register and database of patients with symptomatic haemangioblastomas for the last 15 years was evaluated. The $V H L$ gene was analysed by the SSCP method for all exons and Southern blotting for mutations and deletions of the gene.

Results-141 patients with haemangioblastoma of the CNS were registered. In 81 patients $(57 \%)$ there was a disease predisposing germline mutation including eight novel mutations. Population related calculation of patients from the administrative district of Freiburg disclosed $V H L$ germline mutations in $22 \%$ of the patients with haemangioblastoma. Analysis of mutation carriers for clinical information suggestive of the syndrome showed (1) a positive family history of a brain tumour in $50 \%$, (2) a history for the patient of extracranial manifestations in $36 \%$ (retinal angioma $30 \%$, pheochromocytoma $6 \%$ ), and (3) $19 \%$ presenting with multiple brain tumours when first admitted. By genetic testing of haemangioblastoma patients without any indications of von Hippel-Lindau disease mutation carriers were identified in $14 \%$. Sensitivity of $V H L$ germline testing was $86 \%$.

Conclusions-DNA analysis for $V H L$ germline mutations is clearly superior to clinical information in the diagnosis of von Hippel-Lindau disease. Although the percentage of von Hippel-Lindau disease associated haemangioblastoma decreases after the fourth decade of life and is infrequent in patients without other symptomatic lesions and a negative family history, it is recommended that every patient with CNS haemangioblastoma should be screened for von Hippel-Lindau disease germline mutations. This provides the key information and enables screening for extraneurological tumours of the patients and investigations of the patient's family to ameliorate management of von Hippel-Lindau disease.

(F Neurol Neurosurg Psychiatry 1999;67:758-762)

Keywords: haemangioblastoma; von Hippel-Lindau disease; $V H L$ gene

Haemangioblastomas are histologically benign tumours of the $\mathrm{CNS}^{1}$ but can be life threatening because of large cystic components in the posterior fossa or can cause paraplegia if localised in the spinal canal. Currently, for cerebellar haemangioblastomas the results of treatment are excellent, but relapses or multifocal occurrence may still produce serious complications. Haemangioblastomas occur as sporadic tumours or as part of von Hippel-Lindau disease, an autosomal dominant disorder characterised by benign and malignant tumours predominantly of the retina, kidneys, adrenal glands, pancreas, epididymis, and inner ear in addition to CNS haemangioblastomas. ${ }^{2}$ The diagnosis of von Hippel-Lindau disease is often missed for long periods as (1) additional lesions may be asymptomatic and (2) the syndrome is often not considered in patients with haemangioblastoma of the CNS. An earlier diagnosis, however, is the key for adequate management of such patients and their relatives. Since the VHL tumour suppressor gene was identified in $1993,{ }^{3}$ molecular genetic testing for disease predisposing mutations is possible. Because there are many therapeutic options for the broad range of lesions associated with von Hippel-Lindau disease, molecular genetic testing should be offered to patients at risk; this has been suggested, among others, by the American Society of Clinical Oncology in $1996 .{ }^{4}$

We performed a study of a large series of haemangioblastomas of the CNS to evaluate the impact of molecular genetic testing of the $V H L$ gene for such patients. We also looked for genotype-phenotype correlations, especially regarding the severity of the clinical course.

\section{Methods}

PATIENTS

We established a register of all patients with haemangioblastomas admitted to the department of neurosurgery of our hospital from 1983 to 1998 . We also included such patients operated on by other centres who consulted us 
for genetic testing of the VHL gene. Our systematic survey of each patient included clinical information of intracranial as well as spinal findings, detailed data from ophthalmological, and visceral findings, and an extensive pedigree analysis. Testing for VHL germline mutations and genetic counselling according to the recommendation of the American Society of Clinical Oncology was offered. Our procedure was approved by the ethics committee of the University of Freiburg and our patients gave informed consent. The diagnosis of von Hippel-Lindau disease was established, if the patients had a haemangioblastoma of the CNS and additionally (1) retinal angiomas, renal cell carcinoma, pheochromocytoma, or epididymal cystadenoma; or (2) any first grade relative showing a lesion of von Hippel-Lindau disease; or (3) a positive molecular genetic result. All other patients were classified as having a sporadic haemangioblastoma.

MOLECULAR GENETIC ANALYSES

Genomic DNA was isolated from peripheral blood by standard methods. ${ }^{5}$ Southern blotting was performed to detect large deletions in the VHL gene. Genomic DNA $(7 \mu \mathrm{g})$ was digested with excess Eco RI (Boehringer Mannheim). The fragments were separated in a $0.6 \%$ agarose gel with $1 \times \mathrm{TBE}$ buffer and transferred to a positively charged nylon membrane (Boehringer Mannheim) by capillary blot. Fragments were visualised with the DIG high prime labelling and detection starter kit I (Boehringer Mannheim) according to the supplier. The probes for hybridisation of the Southern blot were made by two sets of primers, one in the very beginning of exon 1 and another set in the 3 'untranslated region of exon 3.

Single strand conformation polymorphism (SSCP) analysis was used to find point mutations, small deletions, or insertions. Four sets of primers were needed to cover all three exons. Polymerase chain reaction (PCR) amplified fragments $(10 \mu \mathrm{l})$ were denatured by adding $15 \mu \mathrm{l}$ denaturing solution (containing $95 \%$ formamide, $10 \mathrm{mM} \mathrm{NaOH}, 0.05 \%$ xylene cyanol, $0.05 \%$ bromophenol blue) and heating to $96^{\circ} \mathrm{C}$ for 3 minutes before chilling on ice. Denatured fragments were separated on a polyacrylamide gel $\left(\mathrm{MDE}^{\mathrm{TM}}\right.$ Gel Solution, FMC Bioproducts, Europe) with $0.5 \times \mathrm{MDE}$ and $0.6 \times \mathrm{TBE}$ buffer according to the manufacturer. After separation at $200 \mathrm{~V}$ for 16 hours the fragments were stained with silver as described elsewhere. ${ }^{6}$ Aberrant bands were cut out of the gel, dissolved in water, and reamplified for sequencing. All mutations were confirmed by sequencing. Mixtures contained 100 ng genomic DNA, $0.2 \mathrm{mM}$ dNTP, $0.5 \mathrm{pmol} / \mu \mathrm{l}$ of each primer, $\mathrm{MgCl}_{2}$, and $0.1 \mathrm{U} / \mu \mathrm{l}$ Taq DNA polymerase (Gibco BRL). PCR conditions and sets of primers have been previously described. ${ }^{7}$

\section{STATISTICS}

For statistical calculations we used the $\chi^{2}$ test for binomial distributions and the Wilcoxon rank sum test for other distributions. Significant differences had $\mathrm{p}$ values $<0.05$.

\section{Results}

REGISTER

Fifty two of the 81 patients operated on in our clinic agreed to molecular genetic testing for germline mutations of the $V H L$ gene and were included in our register. Another 89 patients consulted us for molecular genetic testing. By September 1998 our register included 141 patients with symptomatic haemangioblastiomas of the CNS, who had molecular genetic testing for $V H L$ germline mutations. The age at neurosurgery varied from 11 to 71 (mean 37) years (fig 1). Sex distribution was $53 \%$ female and $47 \%$ male.

The tumours of $63 \%$ of the 141 patients were localised in the cerebellum with a preference of the hemispheres. Five per cent of the haemangioblastomas were brain stem tumours. Thirty two per cent of the haemangioblastomas were found in the spinal canal. Of these, $36 \%$ were localised in the cervical part, $48 \%$ in the thoracic part, and $16 \%$ of the spinal tumours were found in the lumbar area.

\section{GENOTYPES}

In 81 of the 141 patients $(57 \%)$ we detected a germline mutation of the $V H L$ gene. Forty two different mutations were found (table 1). Eight of these mutations are novel and so far not described. ${ }^{8}{ }^{9}$ Considering all mutations, $48 \%$ were of the missense type, $18 \%$ were nonsense mutations, $11 \%$ were intraexonic deletions or insertions, $6 \%$ were splice site mutations, and $16 \%$ were large deletions. Thus $36 \%$ of the mutations are predicted to cause truncation and $16 \%$ deletion of the putative VHL protein.

Population related calculation showed that $22 \%$ of the patients with CNS haemangioblastomas from the area of South Baden had VHL germline mutations. By splitting this into age groups we found that $36 \%$ of the patients under the age of 40 years at diagnosis of the CNS tumour have mutations, whereas $10 \%$ of the patients over 40 years are affected.

Sensitivity of $V H L$ germline testing was $86 \%$ as in addition to the 81 patients with positive tests, 13 patients had von Hippel-Lindau disease according to clinical criteria but were negative when tested by molecular genetics. All patients who were tested positive by molecular genetic analysis also turned out to be positive by clinical criteria.

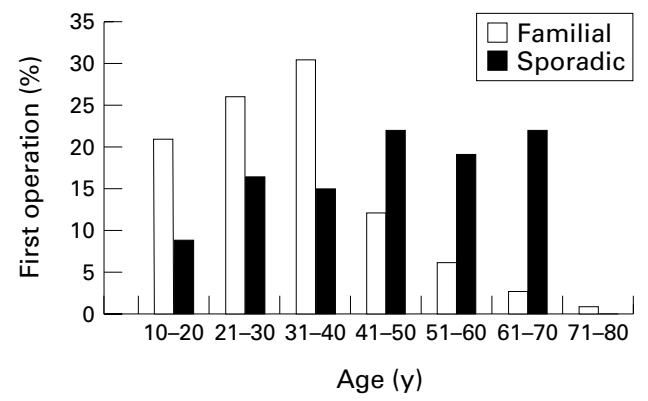

Figure 1 Distribution of age at first neurosurgical operation of patients with familial and sporadic haemangioblastomas in age groups. 
Table 1 von Hippel-Lindau disease germline mutations found in patients with haemangioblastoma of the CNS

\begin{tabular}{|c|c|c|c|c|c|c|}
\hline Nucleotide change & Codon & Protein change & Mutation name & Mutation type & $\begin{array}{l}\text { No of } \\
\text { patients }\end{array}$ & Asymptomatic \\
\hline \multicolumn{7}{|l|}{ Exon 1: } \\
\hline nt $407 \mathrm{C} / \mathrm{T}$ & 65 & Ser $\rightarrow$ Leu & S65L & Missense & 1 & \\
\hline nt $407 \mathrm{C} / \mathrm{G}$ & 65 & Ser $\rightarrow$ Trp & S65W & Missense & 2 & \\
\hline nt $407 \mathrm{C} / \mathrm{A}$ & 65 & Ser $\rightarrow$ stop & S65stop & Nonsense & 1 & \\
\hline nt $434 \mathrm{~T} / \mathrm{G}$ & 74 & $\mathrm{Val} \rightarrow \mathrm{Gly}$ & V74G & Missense & 3 & (1) \\
\hline nt 437 Ins. A & 76 & FS & 437insA & Frameshift & 2 & \\
\hline nt 437 Del.TCT* $^{\star}$ & 76 & Del.Phe & 437 del3 & In-frame del & 2 & \\
\hline nt 443 Ins.TCT & 77 & Ins. Leu & 443 ins 3 & In-frame ins & 1 & \\
\hline nt $446 \mathrm{~A} / \mathrm{G}$ & 78 & Asn $\rightarrow$ Ser & N78S & Missense & 1 & \\
\hline nt $452 \mathrm{G} / \mathrm{A}$ & 80 & Ser $\rightarrow$ Asn & $\mathrm{S} 80 \mathrm{~N}$ & Missense & 1 & \\
\hline nt $454 \mathrm{C} / \mathrm{T}$ & 81 & Pro $\rightarrow$ Ser & P81S & Missense & 1 & \\
\hline nt $475 \mathrm{~T} / \mathrm{A}$ & 88 & Trp $\rightarrow$ Arg & W88R & Missense & 1 & (1) \\
\hline nt $477 \mathrm{G} / \mathrm{C}$ & 88 & Trp $\rightarrow$ Cys & W88C & Missense & 1 & \\
\hline nt $479 \mathrm{~T} / \mathrm{C}$ & 89 & Leu $\rightarrow$ Pro & L89P & Missense & 6 & (2) \\
\hline nt $493 \mathrm{G} / \mathrm{T}$ & 94 & Glu $\rightarrow$ stop & E94stop & Nonsense & 1 & \\
\hline nt $505 \mathrm{~T} / \mathrm{C}$ & 98 & $\mathrm{Tyr} \rightarrow \mathrm{His}$ & $\mathrm{Y} 98 \mathrm{H}$ & Missense & 21 & (13) \\
\hline nt 529 Ins.GCC ${ }^{\star}$ & 106 & Ins. Arg & 529ins3 & In-frame ins & 1 & \\
\hline nt $544 \mathrm{~A} / \mathrm{C}^{\star}$ & 111 & Ser $\rightarrow$ Arg & S111R & Missense & 1 & \\
\hline nt $553+1 \mathrm{G} / \mathrm{A}^{\star}$ & & splice defect & $553+1 \mathrm{G} / \mathrm{A}$ & Splice defect & 1 & \\
\hline \multicolumn{7}{|l|}{ Exon 2: } \\
\hline nt $557 \mathrm{~A} / \mathrm{G}$ & 115 & His $\rightarrow$ Arg & H115R & Missense & 2 & (1) \\
\hline nt 559 Ins. $T^{\star}$ & 116 & FS & 559insT & Frameshift & 1 & \\
\hline nt 597 Del. T & 128 & FS & $597 \mathrm{delT}$ & Frameshift & 1 & \\
\hline nt $607 \mathrm{C} / \mathrm{T}$ & 132 & Gln $\rightarrow$ stop & Q132stop & Nonsense & 1 & \\
\hline nt $608 \mathrm{~A} / \mathrm{C}$ & 132 & Gln $\rightarrow$ Pro & Q132P & Missense & 2 & (1) \\
\hline nt $620 \mathrm{~T} / \mathrm{C}$ & 136 & Phe $\rightarrow$ Ser & F136S & Missense & 1 & \\
\hline nt $620 \mathrm{~T} / \mathrm{G}$ & 136 & Phe $\rightarrow$ Cys & F136C & Missense & 1 & \\
\hline nt $646 \mathrm{C} / \mathrm{T}^{\star}$ & 145 & Glu $\rightarrow$ stop & E145stop & Nonsense & 3 & \\
\hline nt $665 \mathrm{~T} / \mathrm{C}^{\star}$ & 151 & $\mathrm{Ile} \rightarrow \mathrm{Thr}$ & I151T & Missense & 2 & \\
\hline nt $676+1 \mathrm{G} / \mathrm{C}$ & & Splice defect & $676+1 \mathrm{G} / \mathrm{C}$ & Splice defect & 2 & \\
\hline nt $676+2 \mathrm{C} / \mathrm{T}$ & & Splice defect & $676+2 \mathrm{C} / \mathrm{T}$ & Splice defect & 1 & \\
\hline \multicolumn{7}{|l|}{ Exon 3: } \\
\hline nt $677-2 \mathrm{~A} / \mathrm{G}$ & & Splice defect & $677-2 \mathrm{~A} / \mathrm{G}$ & Splice defect & 1 & \\
\hline nt $694 \mathrm{C} / \mathrm{G}$ & 161 & $\mathrm{Arg} \rightarrow \mathrm{Gly}$ & R161G & Missense & 1 & \\
\hline nt $694 \mathrm{C} / \mathrm{T}$ & 161 & Arg $\rightarrow$ stop & R161stop & Nonsense & 4 & \\
\hline nt $695 \mathrm{G} / \mathrm{C}$ & 161 & Arg $\rightarrow$ Pro & R161P & Missense & 1 & \\
\hline nt $695 \mathrm{G} / \mathrm{A}$ & 161 & $\mathrm{Arg} \rightarrow \mathrm{Gln}$ & R161Q & Missense & 1 & \\
\hline nt $699 \mathrm{C} / \mathrm{G}$ & 162 & Cys $\rightarrow$ Trp & $\mathrm{C} 162 \mathrm{~W}$ & Missense & 1 & \\
\hline nt $703 \mathrm{C} / \mathrm{T}$ & 164 & Gln $\rightarrow$ stop & Q164stop & Nonsense & 1 & \\
\hline nt $712 \mathrm{C} / \mathrm{T}$ & 167 & Arg $\rightarrow$ Trp & R167W & Missense & 5 & (1) \\
\hline nt $713 \mathrm{G} / \mathrm{A}$ & 167 & $\operatorname{Arg} \rightarrow \mathrm{Gln}$ & R167Q & Missense & 1 & \\
\hline nt $746 \mathrm{~T} / \mathrm{A}$ & 178 & $\mathrm{Leu} \rightarrow \mathrm{Gln}$ & L178Q & Missense & 2 & (1) \\
\hline nt $746 \mathrm{~T} / \mathrm{C}$ & 178 & Leu $\rightarrow$ Pro & L178P & Missense & 1 & \\
\hline nt $761 \mathrm{C} / \mathrm{A}$ & 183 & Ser $\rightarrow$ stop & S183stop & Nonsense & 4 & \\
\hline nt 794 Del.GT* & 194 & FS & 794del2 & Frameshift & 1 & \\
\hline \multicolumn{7}{|l|}{ Large deletions: } \\
\hline $2 \mathrm{~kb}$ Deletion & & & & Deletion & 5 & \\
\hline $3 \mathrm{~kb}$ Deletion & & & & Deletion & 1 & \\
\hline $10 \mathrm{~kb}$ Deletion & & & & Deletion & 5 & \\
\hline $11 \mathrm{~kb}$ Deletion & & & & Deletion & 2 & \\
\hline
\end{tabular}

A germline mutation of the VHL gene was found in 81 of the 141 patients with primary symptomatic haemangioblastoma. Additionally we could find mutations in 21 patients with primary asymptomatic tumours. Forty two different mutations were found. $\mathrm{nt}=$ nucleotide; ins=insertion; del=deletion; FS=frameshift; ${ }^{\star}$ novel mutations.

PHENOTYPES

Evaluation of the clinical characteristics of the 81 patients with primary symptomatic haemangioblastoma and $V H L$ germline mutations are as follows. Previous symptomatic VHL associated lesions were found in $36 \%$ of these patients: Thirty per cent had retinal angiomas, $6 \%$ had pheochromocytomas, but no one had had a previous operation for kidney cancer. A family history for "brain tumour" was found in $50 \%$, and $84 \%$ had a family history for retinal angiomas, kidney cancer, pheochromocytoma, or epididymal tumours. Asymptomatic VHL associated lesions were found in $70 \%$ by clinical screening. Of these, $78 \%$ had retinal angiomas, $18 \%$ had pheochromocytomas, and $16 \%$ had kidney cancer.

Multiple haemangioblastomas have been documented in $19 \%$ of our patients with von Hippel-Lindau disease when first admitted for neurological symptoms. However, by complete neuroimaging and follow up $73 \%$ of the patients had more than one haemangioblastoma (fig 2).
Sixty six of our patients had no clinical hints for von Hippel-Lindau disease when first admitted for neurological symptoms. They had only one CNS tumour, a negative family history for von Hippel-Lindau disease, and no other symptomatic von Hippel-Lindau disease associated lesion, not including the results of ophthalmological and radiological screening.

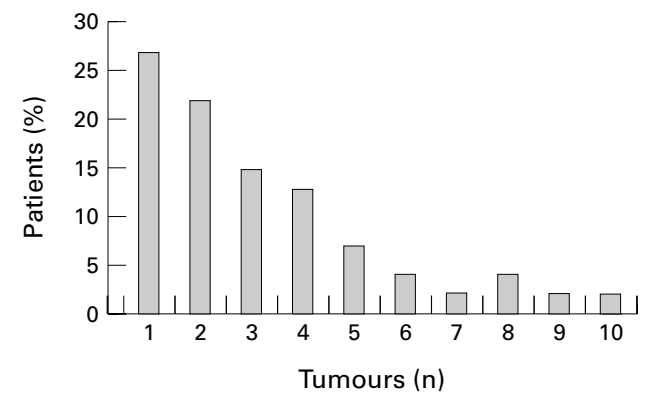

Figure 2 Multiple occurrence of familial haemangioblastomas. Although most patients have multiple tumours, $27 \%$ of the familial cases present with a solitary haemangioblastoma. 
Nine of these patients (14\%) including two older than 60 years were tested positive by genetic screening and hereby identified as patients with von Hippel-Lindau disease. When clinically screened, three of these nine subjects did not show extra CNS lesions and only three turned out to have a positive family history.

GENOTYPE-PHENOTYPE CORRELATION

Patients with sporadic versus von HippelLindau disease associated haemangioblastomas showed the following significant differences. Thirty five per cent of haemangioblastomas associated with von HippelLindau disease were localised in the spinal canal versus $20 \%$ of the sporadic tumours $(p<0.02)$. Patients with haemangioblastomas associated with von Hippel-Lindau disease were 11 years younger than patients with sporadic haemangioblastomas $(p<0.001$, fig 1).

In patients with haemangioblastomas associated with von Hippel-Lindau disease we compared severity of the neurological disease with types of VHL germline mutation subdividing mutations predicted to produce a full length von Hippel-Lindau disease protein (F-type) and mutations causing truncation or deletion of the von Hippel-Lindau disease protein (T-type). Patients with T-type mutations developed more multiple tumours than patients with F-type mutations $(78 \%$ v $57 \%, \mathrm{p}<0.02)$ and had more frequent multiple operations $(56 \% v$ $23 \%, \mathrm{p}<0.05$ )

\section{ASYMPTOMATIC HAEMANGIOBLASTOMAS}

Once the VHL germline mutation was determined in a patient with CNS haemangioblastoma, genetic family screening was performed followed by neuroimaging of mutation carriers. Thus 82 asymptomatic haemangioblastomas have been detected in 21 subjects. The localisation of the tumours was in three cases the posterior fossa, in 10 cases the spinal canal, and in eight cases both posterior fossa and spinal canal.

costs

We evaluated the costs of the clinical and the molecular genetic screening programme. The clinical screening programme as listed in table $2^{10}$ costs 2570 Euro and is performed annually. Molecular genetic screening costs 960 Euro or, if sequencing is necessary, 1070 Euro. Once a mutation carrier is identified, the genetic screening costs 290 Euro/family member.

Table 2 Clinical examination programme for potential patients with von Hippel-Lindau disease

- Gd enhanced MR imaging of the brain

- Gd enhanced MR imaging of the spinal canal

- MR imaging of the abdomen

- Ophthalmological examination

- Fluorescein angiography of the retina

- Twenty four hour urinary catecholamine excretion

This programme should be performed annually in patients with von Hippel-Lindau disease and there is currently no consensus for a larger interval for any of these investigations in a given germline mutation.

\section{Conclusions}

Haemangioblastomas of the CNS occur as a sporadic entity or as part of von Hippel-Lindau disease. Although management of sporadic tumours is standardised and does not cause major problems, familial haemangioblastomas need a completely different clinical approach concerning the patients and their families. However, the diagnosis still causes difficulties and is often missed, because the syndrome varies strongly in severity, number of lesions, and number of affected organs, ${ }^{11}$ and pedigree analysis is often not performed carefully enough.

Because haemangioblastoma is the most common preceding manifestation in von Hippel-Lindau disease, ${ }^{12}$ the safe molecular genetic analysis of the VHL gene in patients with haemangioblastomas plays a key part in the diagnosis of the disease. On average the diagnosis of von Hippel-Lindau disease is made 4.5 years after the onset of symptoms. ${ }^{12}$ Early detection of associated lesions ${ }^{13}$ and consequent follow up, however, is essential for adequate management of this cancer syndrome.

As multiple occurrence of haemangioblastomas was only found in one sporadic case, whereas $64 \%$ of the familial cases had more than one haemangioblastoma by complete neuroimaging and follow up, multiple occurrence is a good index for von Hippel-Lindau disease. But only $19 \%$ of our patients with the disease showed multiple haemangioblastomas when first admitted for neurological symptoms, and only $36 \%$ had previous symptomatic von Hippel-Lindau disease associated lesions, which makes the diagnosis of the disease based on clinical criteria unsafe in patients with haemangioblastomas of the CNS.

Pedigree analysis, if very carefully performed, had a higher sensitivity in identifying patients with von Hippel-Lindau disease (50\% for "brain tumours", $84 \%$ for other manifestations).

The highest sensitivity, however, related to molecular genetic analysis of the VHL gene. We detected VHL mutations in $86 \%$ of the patients, who turned out to be affected with von Hippel-Lindau disease. Sensitivity may be increased by improvement of molecular genetic methodology as a first report presents a rate of $99 \% .{ }^{14}$ As all patients who were tested positive by molecular genetic analysis turned out also to be positive by clinical criteria, it is a very secure method.

In the subsequent clinical screening programme for patients with von Hippel-Lindau disease (table 2), primary asymptomatic lesions of other organs were found in $70 \%$ of the patients. These lesions were controlled by follow up screening, and, if necessary, operated on at an early stage. By molecular genetic family screening and subsequent neuroimaging of mutation carriers, we furthermore detected 82 primary asymptomatic haemangioblastomas in 21 apparently unaffected patients, which could be controlled and removed at an early stage before symptoms develop. By contrast, $50 \%$ of the primary symptomatic patients are admitted for emergency treatment. ${ }^{15}$ 
Looking for genotype-phenotype correlations showed that patients with mutations causing truncation or deletion of the von Hippel-Lindau disease protein (T-type) developed more frequent multiple tumours and had more frequent multiple operations than patients with mutations predicted to produce a full length von Hippel-Lindau disease protein (F-type). These results suggest that T-type mutations induce a more severe course, and might have influence on the clinical management. To optimise it, international cooperation is needed to extend mutational data for tumour growth, size, complications, location, and age of manifestation.

Our data show that mutation analysis is of striking importance for the clinical course and management. For patients who present with a primary symptomatic solitary haemangioblastoma, negative family history, and no other known manifestation of the von Hippel-Lindau disease complex, molecular genetic analysis is the only safe instrument of diagnosis. In this apparently unaffected group without clinical hints for von Hippel-Lindau disease we found mutations of the VHL gene in nine of $66(14 \%)$ of the patients, two of them older than 60 years, three of them even after clinical screening without extra CNS lesions, and three still with a negative family history. Considering the fact that detailed information about the family history is often not easily available, the percentage of affected patients in this group will be higher in clinical use. This justifies DNA testing in patients without clinical indications in all age groups.

For members of families with von HippelLindau disease who show no lesion of the disease complex molecular genetic testing is the only adequate method, as complete clinical screening of all family members would be overinvestigation and results according to our experience in a low compliance. Moreover, it is too expensive and too insecure, especially for young family members, who are unlikely to show a manifestation. It is urgently necessary to identify mutation carriers in the family by molecular genetic analysis.

Even for patients, who are obviously affected with von Hippel-Lindau disease, mutation analysis is still advisable, because (1) the result will confirm the clinical diagnosis and (2) once mutation specific data will be extended, we may be able to characterise the specific aggressivity, which can have consequences on the management of the patient.

As our results show, the percentage of von Hippel-Lindau disease associated haemangioblastomas decreases after the fourth decade of life $(10 \% v 36 \%)$, but is still high and a clear basis that genetic testing for von HippelLindau disease germline mutation has to be recommended in this age also.

The fact that $64 \%$ of the patients agreed to molecular genetic testing after being informed about its impact shows the high acceptance on the part of the patients.

Mutations of the VHL gene were not rare in our patient group $(22 \%)$ and their knowledge is important to clinicians and basic scientists. Our results are in good agreement with the recommendations of the American Society of Clinical Oncology, that genetic counselling and testing should be offered to patients at risk and more responsibly integrated into the practice of clinical and preventive oncology. ${ }^{4}$ Surely all patients with haemangioblastomas of the CNS should be considered as patients of risk in this context.

We conclude that DNA analysis for VHL germline mutations is clearly superior to clinical information in diagnosis of von Hippel-Lindau disease. It is safe, inexpensive, and easily available. Although the percentage of associated haemangioblastoma decreases after the fourth decade of life and is infrequent in patients without other symptomatic lesions and a negative family history, we recommend that every patient with CNS haemangioblastoma should be screened for von Hippel-Lindau disease germline mutations. This provides the key information and enables screening for extraneurological tumours of the patients and investigations of the patient's family to ameliorate management of von Hippel-Lindau disease.

This work was supported by a grant from the Center of Clinical Research of the University of Freiburg, Germany.

1 Rubinstein LJ. Tumors of the central nervous system. Atlas of tumor pathology, series 2, fascicle 6. Washington, DC: Armed Forces Institute of Pathology, 1972:235.

2 Neumann HPH, Lips CJM, Hsia YE, Zbar B. Von HippelNeumann HPH, Lips CJM, Hsia YE, Zbar B. V
Lindau syndrome. Brain Pathol 1995;5:181-93.

3 Latif F, Tory K, Gnarra J, et al. Identification of the von Hippel-Lindau disease tumor suppressor gene. Science 1993;260:1317-20.

4 The American Society of Clinical Oncology. Genetic testing for cancer susceptibility. F Clin Oncol 1996;14:1730-6.

5 Sambrook J, Fritsch EF, Maniatis T. Molecular cloning. Cold Spring Harbor, NY: Cold Spring Harbor Labotatory Press, 1989.

6 Budowle B, Chakraborty R, Giusti AM, et al. Analysis of the VNTR locus D1S 80 by PCR followed by high resolution PAGE. Am f Hum Genet 1991;48:137-44

7 Gnarra JR, Tory K, Weng Y, et al. Mutations of the $V H L$ tumor suppressor gene in renal carcinoma. Nat Genet 1994;7:85-90.

8 Neumann HPH, Bender BU. Genotype-phenotype correlations in von Hippel-Lindau disease. 7 Intern Med 1998;243:541-5.

9 Beroud C, Joly D, Gallou C, et al. Software and database for the analysis of mutations in the VHL gene. Nucleic Acids Res 1998;26:256-8.

10 Neumann HPH. Basic criteria for clinical diagnosis and genetic counselling in von Hippel-Lindau syndrome. $\mathcal{F}$ Vasc genetic counselling in

11 Neumann HPH. Von Hippel-Lindau-Syndrom Unterschätzt und häufig verkannt. Deutsches Ärzteblatt 1993;11: $786-93$

12 Maddock IR, Moran A, Maher ER, et al. A genetic register for von Hippel-Lindau disease. F Med Genet 1996;33:1207.

13 Lamiell JM, Salazar FG, Hsia YE. Von Hippel-Lindau disease affecting 43 members of a single kindread. Medicine (Baltimore) 1989;68:1-29.

14 Stolle C, Glenn G, Zbar B, et al. Improved detection of germline mutations in the von Hippel-Lindau disease germline mutations in the von Hippel-Lindau disea

15 Neumann HPH, Eggert HR, Weigel K, et al. Hemangioblastomas of the central nervous system. F Neurosurg 1989;70: tomas of 